\title{
Antenna calibration using channel prediction for time-varying channels
}

\author{
Yamin SONG, Xia LEI*, Zhaofu KONG, Binhong DONG \\ National Key Laboratory of Science and Technology on Communications, University of Electronic Science and Technology of \\ China, Chengdu 610054, China
}

\begin{abstract}
Traditional antenna calibration methods for time division duplex (TDD) systems assume that the free-space channel remains the same during calibration, which is unreasonable under the high-speed rail and other time-varying channel scenarios, and will cause calibration error due to time variability. This paper proposes an antenna calibration method for time-varying channels. In the proposed method, the transceiver first sequentially sends a pilot signal to obtain equivalent downlink and uplink channel responses. Then, by predicting the downlink (uplink) channel response fed back from the receiver using the channel prediction algorithm, the transmitter obtains the channel response corresponding to the channel response on uplink (downlink). Finally, the transmitter calculates the transmission calibration factor through the prediction value. Compared with the traditional antenna calibration method, this method can improve the accuracy of the calibration factor. Simulation results show that the performance degradation of antenna calibration can be caused by time-varying channels and the proposed method can well compensate for the performance loss and significantly improve the antenna calibration performance for time-varying channels.
\end{abstract}

Key words: TDD; channel reciprocity; time-varying channel; channel prediction; antenna calibration

(C) 2012 JMT. All rights reserved.

\section{Introduction}

$\mathrm{T}$ he known channel information of the transmission side can improve the performance of wireless communication systems [1-2]. In an ideal time division duplex (TDD) system, as the downlink channel and uplink channel occupy the same frequency band, the transmitter can obtain downlink (uplink) channel information from uplink (downlink) channel measurements, i.e. channel reciprocity. Therefore, without any feedback information, the transmitter in TDD systems can easily obtain channel information for effective transmit beamforming and pre-equalization [3].

However, in practical TDD systems, the communication channel includes not only the actual free-space channel, but also the antenna, radio frequency (RF) amplifier, filter, and analog-to-digital converter (ADC) [4-6]. For different transceivers, the responses of the above devices are not equal and this will cause channel reciprocity loss of the actual equivalent downlink and uplink channels [7].

To date, related studies have yielded many results. In

Received Oct. 15, 2012; revision accepted Nov. 25, 2012

*Corresponding author. Tel.: +86-28-61830319

E-mail: leixia@uestc.edu.cn (X. LEI)

(C) 2012 JMT. All rights reserved

doi: 10.3969/j.issn.2095-087X.2012.04.004
[8] and [9], the response of analog devices was thought to be related with the external environment, such as temperature, time, and power; mathematical models of the analog devices' response that change with environmental parameters were established. An absolute calibration that requires an external reference source with tight requirements was proposed in [10], and a relative calibration that depends on a calibration phase to estimate the difference between uplink and downlink was proposed in [11-12]. Compared to the absolute calibration method, the relative calibration method is more suitable for practical applications because of lower price and no feedback.

However, the above methods assume that free-space channel remains the same during calibration, which is unreasonable under the high-speed rail and other timevarying channel scenarios. For time-varying channels, even though the calibration period is very short, the freespace channel is still changing. When the antenna calibration methods above are used for time-varying channels, loss of system performance is expected because of the calibration error caused by time variability of channels.

In this paper, we verify by analysis and simulation that the performance deterioration can be caused by time-varying channels in TDD-OFDM (orthogonal frequency division multiplexing) pre-equalization system in comparison with that in TDD-MIMO (multiple-input multiple-out-put) pre-coding system. A relative antenna 
calibration method is proposed using a channel prediction for time-varying channels. Simulation results show the effectiveness of the proposed algorithm.

The rest of the paper is organized as follows. In Section 2, we describe the signal transmission model and the traditional antenna calibration model in TDDOFDM system. Then we analyze the performance of the traditional antenna calibration for time-varying channels and propose our algorithm in Section 3. Simulation results are presented in Section 4. Finally, Section 5 states the conclusion.

\section{System model}

In this section, we describe the signal transmission model and the traditional antenna calibration model in the TDD-OFDM system.

\subsection{Signal model}

Fig. 1 shows the signal transmission model in TDDOFDM system. The data bit at base station (BS) is modulated into complex symbols which are then preequalized on each sub-carrier. After OFDM modulation, the OFDM symbols are up-converted and amplified by RF front end and transmitted to the free-space. At the user equipment (UE), the received signal is sent to an OFDM demodulator after down-conversion and then demodulated to the output.

The RF front-end model at BS and UE is shown in Fig. 2, where the RF front-end response at the transmitter and receiver are independent; $g^{(\mathrm{UL})}$ and $g^{(\mathrm{DL})}$ denote the uplink and downlink free-space channel response, respectively; $T_{\mathrm{BS}}$ and $R_{\mathrm{BS}}$ denote the transmitting antenna RF front-end response and the receiving antenna
$\mathrm{RF}$ front-end response at $\mathrm{BS}$, respectively, while $T_{\mathrm{UE}}$ and $R_{\mathrm{UE}}$ denote the counterparts at UE; ADC and DAC denote analog to digital converter and digital to analog converter, respectively. According to the electromagnetic theory, a free-space channel is reciprocal, .i.e. $g^{(\mathrm{DL})}=g^{(\mathrm{UL})}[11,13]$.

Let $h_{\text {pra }}^{(\mathrm{UL})}$ and $h_{\text {pra }}^{(\mathrm{DL})}$ denote the equivalent channel on uplink and downlink, respectively, when considering the impact of antenna RF front-end. Then we can get from Fig. 2 that [11]

$$
\left.\begin{array}{l}
h_{\text {pra }}^{(\mathrm{UL})}=T_{\mathrm{UE}} g^{(\mathrm{UL})} R_{\mathrm{BS}}, \\
h_{\text {pra }}^{(\mathrm{DL})}=T_{\mathrm{BS}} g^{(\mathrm{DL})} R_{\mathrm{UE}} \cdot
\end{array}\right\}
$$

We can see from Eq. (1) that $h_{\text {pra }}^{(\mathrm{UL})} \neq h_{\text {pra }}^{(\mathrm{DL})}$ because of independence of the RF front ends at BS and UE, which means non-reciprocity of equivalent channel on uplink and downlink. In order to compensate the channel nonreciprocity, antenna calibration is used to find out differences between RF front ends at BS and UE, and compensate the mismatches of the equivalent channel at the transmitter by the calibration factor. Let $c_{\mathrm{BS}}$ and $c_{\mathrm{UE}}$ in Fig. 2 denote transmission calibration factors at BS and UE, respectively.

\subsection{Antenna calibration model}

As mentioned above, there are two typical antennal calibration methods: absolute calibration and relative calibration. The absolute method calibrates the RF front end using additional hardware, and will increase the cost and design complexity. The relative calibration, without

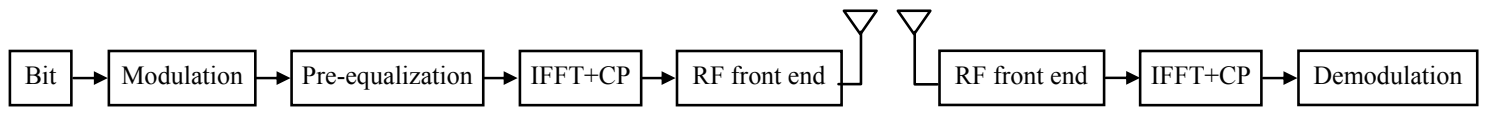

Fig. 1 Signal transmission model in TDD-OFDM system

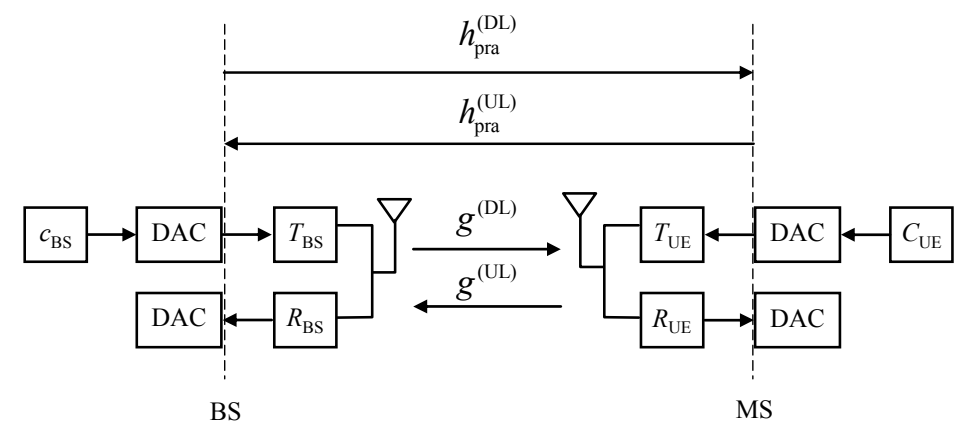

Fig. 2 RF front-end model 
changing the hardware, finds out differences between $\mathrm{RF}$ front ends at BS and UE and compensates for them using a software algorithm. Therefore, the relative calibration method is more suitable for practical applications [14-16].

The calibration factor $c_{\mathrm{BS}}$ and $c_{\mathrm{UE}}$ through relative calibration is obtained in the following steps [16]:

(1) Initialize $c_{\mathrm{BS}}=c_{\mathrm{UE}}=1$.

(2) BS sends calibration (pilot) signal to UE. UE receives the signal and measures the downlink equivalent channel response given by

$h_{\mathrm{pra}}^{(\mathrm{DL})}=T_{\mathrm{BS}} g^{(\mathrm{DL})} R_{\mathrm{UE}}$.

(3) UE feeds back the downlink equivalent channel response and at the same time sends calibration (pilot) signal to $\mathrm{BS}$.

(4) BS measures the uplink equivalent channel response given by $h_{\mathrm{pra}}^{(\mathrm{UL})}=T_{\mathrm{UE}} g^{(\mathrm{UL})} R_{\mathrm{BS}}$, and calculates the calibration factor given by

$$
c_{\mathrm{BS}}=\frac{T_{\mathrm{UE}} g^{(\mathrm{UL})} R_{\mathrm{BS}}}{T_{\mathrm{BS}} g^{(\mathrm{DL})} R_{\mathrm{UE}}} .
$$

(5) The calibration phase is over.

During the calibration, the power of the calibration signal is required to be the same as that of data signal because RF front-end response is dependent on signal power. Hence, for channel reciprocity during data signal transmission, the power of the two signals is required to be the same.

To form the calibration process, free-space channels on uplink and downlink are assumed reciprocal during calibration, and the relative calibration method can eliminate the influence of free-space channel to obtain the calibration factor $c_{\mathrm{BS}}=T_{\mathrm{UE}} R_{\mathrm{BS}} /\left(T_{\mathrm{BS}} R_{\mathrm{UE}}\right)$. In practical systems, the difference between $T_{\mathrm{BS}}$ and $R_{\mathrm{BS}}$ is important. Therefore, we define the mismatch parameters as:

$$
\left.\begin{array}{l}
E_{\mathrm{BS}}=\frac{T_{\mathrm{BS}}}{R_{\mathrm{BS}}}=A_{\mathrm{BS}} \exp \left(\mathrm{j} \varphi_{\mathrm{BS}}\right), \\
E_{\mathrm{UE}}=\frac{T_{\mathrm{UE}}}{R_{\mathrm{UE}}}=A_{\mathrm{UE}} \exp \left(\mathrm{j} \varphi_{\mathrm{UE}}\right),
\end{array}\right\}
$$

where $A$ and $\varphi$ denote the magnitude and phase of the mismatch parameter, respectively. The mismatch parameters at BS and UE can be assumed as independent and identically distributed random variables [11]. In this paper, the mismatch parameter model proposed in [17] is used. The magnitude $A$ obeys a logarithmic normal distribution, i.e., $10 \lg A \sim N\left(0, \sigma^{2}\right)$, and the phase $\varphi$ obeys a uniform distribution on the interval $[-\theta, \theta]$.
However, under the high-speed rail and other timevarying channel scenarios, free-space channel is no longer constant even though the calibration period is very short, so it cannot be assumed as reciprocal, i.e. $g^{(\mathrm{DL})} \neq g^{(\mathrm{UL})}$. Therefore, the above traditional antenna calibration will cause calibration error and hence decrease the system performance for time-varying channels.

\section{Antenna calibration using channel pre- diction for time-varying channels}

In this section, we first analyze the performance of traditional antenna calibration for time-varying channels, and then propose our algorithm.

For time-varying channels, the traditional antenna calibration can cause calibration error, which will incur loss in channel reciprocity on uplink and downlink. Then the pre-equalization factor for downlink transmission calculated from the channel measurement on the uplink will mismatch the real downlink channel and cause performance degradation.

Before data signal transmission, antenna calibration is implemented to ensure channel reciprocity. The RF frontend response is time-varying slowly, so it is reasonable to assume a constant front-end response during a calibration or data signal transmission [7]. Thus, the calibration factors are constant once they are determined after calibration. For simplicity, let $g_{\text {cal }}^{(\mathrm{UL})}$ and $g_{\text {cal }}^{(\mathrm{DL})}$ denote the freespace channel response during calibration on uplink and downlink, respectively; $h_{\mathrm{tr}}^{(\mathrm{UL})}$ and $h_{\mathrm{tr}}^{(\mathrm{DL})}$ denote the freespace channel response during data signal transmission on uplink and downlink, respectively.

The free-space channel is assumed to be frequencyflat. From Section 2, we can obtain

$$
c_{\mathrm{BS}}=\frac{T_{\mathrm{UE}} g_{\mathrm{cal}}^{(\mathrm{UL})} R_{\mathrm{BS}}}{T_{\mathrm{BS}} g_{\text {cal }}^{(\mathrm{DL})} R_{\mathrm{UE}}},
$$

where $g_{\text {cal }}^{(\mathrm{UL})} \neq g_{\text {cal }}^{(\mathrm{DL})}$. After calibration, data transmission begins. Then we can obtain the equivalent channel response on uplink and downlink as follows:

$$
\left.\begin{array}{l}
h_{\mathrm{e}}^{(\mathrm{UL})}=T_{\mathrm{UE}} h_{\mathrm{tr}}^{(\mathrm{UL})} R_{\mathrm{BS}}, \\
h_{\mathrm{e}}^{(\mathrm{DL})}=c_{\mathrm{BS}} T_{\mathrm{BS}} h_{\mathrm{tr}}^{(\mathrm{DL})} R_{\mathrm{UE}}
\end{array}\right\}
$$

Assume that the data symbol transmitted on the $k$ th subcarrier is $d_{k}$, and the pre-equalization factor is $\lambda_{k}=1 / h_{\mathrm{e}}^{(\mathrm{UL})}$. By the data transmission model described in Section 2, we can obtain the received data $z_{k}$ on the $k$ th subcarrier as 


$$
\begin{aligned}
& z_{k}=d_{k} \lambda_{k} h_{\mathrm{e}}^{(\mathrm{DL})}+n_{k}= \\
& h_{\mathrm{e}}^{(\mathrm{DL})} \frac{1}{h_{\mathrm{e}}^{(\mathrm{UL})}} d_{k}+n_{k}=\frac{g_{\mathrm{cal}}^{(\mathrm{UL})}}{g_{\text {cal }}^{(\mathrm{DL})}} \frac{h_{\mathrm{tr}}^{(\mathrm{DL})}}{h_{\mathrm{tr}}^{(\mathrm{DL})}} d_{k}+n_{k},
\end{aligned}
$$

where $n_{k}$ denotes the additive white Gaussian noise on the $k$ th subcarrier. From Eq. (5), we can see that the time-varying channel can cause two interferences: (1) interference caused by calibration error, and (2) interference caused by mismatch between the real channel on uplink and downlink during data transmission. Therefore, for time-varying channel, pre-equalization using channel prediction is proposed to eliminate the interference caused by mismatch between channels on uplink and downlink during data transmission [18]. In this paper, we apply channel prediction into antenna calibration to eliminate the interference caused by calibration error during calibration.

Fig. 3 shows the TDD frame structure. The transmitter knows the channel response of the previous time. Then according to the channel model, it can predict the channel response of the current time, namely, channel prediction. Using the frame structure shown in Fig. 3, the antenna calibration method based on channel prediction for timevarying channel can be described as follows:

(1) Initialize $c_{\mathrm{BS}}=c_{\mathrm{UE}}=1$.

(2) BS sends calibration (pilot) signal to UE. UE receives the signal and measures the downlink equivalent channel given by $h_{\mathrm{pra}}^{(\mathrm{DL})}=T_{\mathrm{BS}} g^{(\mathrm{DL})} R_{\mathrm{UE}}$.

(3) UE feeds back the downlink equivalent channel response and at the same time sends calibration (pilot) signal to BS.

(4) BS predicts the channel response through the downlink equivalent channel response fed back form UE, and gets the prediction value $\tilde{h}_{\text {pra }}^{(\mathrm{DL})}$.

(5) BS measures the uplink equivalent channel, and calculates $c_{\mathrm{BS}}=h_{\mathrm{pra}}^{(\mathrm{UL})} / \tilde{h}_{\mathrm{pra}}^{(\mathrm{DL})}$.

(6) The calibration phase is over.

In contrast to the traditional antenna calibration method in Section 2, we find the core of the algorithm is step (4), i.e. prediction followed by calibration.

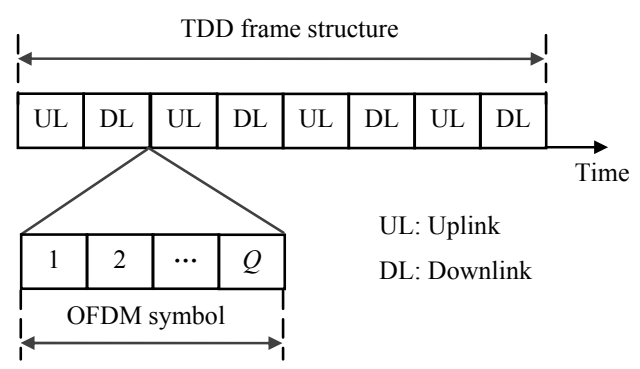

Fig. 3 TDD frame structure
For time-varying channel conditions, the performance loss of the traditional antenna calibration method is caused by the time-varying channel, especially when the channel changes dramatically. The uplink and downlink channels are obviously different, and the error is large. The performance of the proposed algorithm is related to the performance of the channel prediction algorithm used, and the performance loss is caused by the prediction error. The results of previous studies have demonstrated that the prediction error is less than the error caused by the time-varying channel, which is the basis of the channel prediction algorithm research. Therefore, the performance of the proposed algorithm is superior to that of the traditional antenna calibration algorithm for time-varying channel conditions.

Currently, channel prediction research on timevarying channels focuses on linear prediction algorithms, such as the linear dynamic autoregressive (AR) model [19-20]. However, due to the time-varying channel with very complex non-linear characteristics, introduction of the nonlinear prediction algorithm is also the growing concern of researchers. In this paper, a nonlinear prediction algorithm based on least-squares support vector machines (LS-SVMs) is used [21-22]. Using the frame structure shown in Fig. 3, the channel prediction algorithm can be described as follows [22]:

(1) BS receives the downlink equivalent channel response $h_{\mathrm{DL}(q)}(q=1,2, \cdots, Q)$ fed back from UE, and transforms them to $N$ training sequences $\left\{\boldsymbol{x}_{n}, y_{n}\right\}_{n=1}^{N}$, where

$$
\begin{aligned}
\boldsymbol{x}_{n}= & \left(h_{\mathrm{DL}(n)}, h_{\mathrm{DL}(n+1)}, \cdots, h_{\mathrm{DL}(n+m-1)}\right), y_{n}=h_{\mathrm{DL}(n+m)}, \\
\boldsymbol{x}_{n} & \in \mathbf{R}^{m}, y_{n} \in \mathbf{R} .
\end{aligned}
$$

(2) The non-linear mapping function $\varphi(\cdot)$ maps the training sequences $\left\{\boldsymbol{x}_{n}, y_{n}\right\}_{n=1}^{N}$ to a higher dimensional space, i.e. $\varphi(\cdot): \mathbf{R}^{m} \rightarrow \mathbf{R}^{c}$. In this space, the linear optimal regression function can be constructed as

$$
f(\boldsymbol{x})=\boldsymbol{w}^{\mathrm{T}} \varphi(\boldsymbol{x})+b,
$$

where $\boldsymbol{w} \in \mathbf{R}^{c}$ is the weight vector and $b \in \mathbf{R}$ is the deviation. Substituting the training sequence $\left\{\boldsymbol{x}_{n}, y_{n}\right\}_{n=1}^{N}$ into Eq. (5), we obtain

$$
y_{n}=f\left(\boldsymbol{x}_{n}\right)=\boldsymbol{w}^{\mathrm{T}} \varphi\left(\boldsymbol{x}_{\boldsymbol{n}}\right)+b .
$$

(3) LS-SVM is used to transform the regression problem into the optimal planning problem. Thus, the optimal regression function can be obtained as

$$
f(\boldsymbol{x})=\sum_{n=1}^{N} \alpha_{n} K\left(\boldsymbol{x}_{n}, \boldsymbol{x}\right)+b,
$$

where parameters $\alpha_{n}$ and $b$ can be obtained by solving Eq. (9) [22]: 


$$
\left[\begin{array}{cccc}
0 & 1 & \cdots & 1 \\
1 & K\left(\boldsymbol{x}_{1}, \boldsymbol{x}_{1}\right)+1 / \gamma & \cdots & K\left(\boldsymbol{x}_{1}, \boldsymbol{x}_{N}\right) \\
\vdots & \vdots & \ddots & \vdots \\
1 & K\left(\boldsymbol{x}_{N}, \boldsymbol{x}_{1}\right) & \cdots & K\left(\boldsymbol{x}_{N}, \boldsymbol{x}_{N}\right)+1 / \gamma
\end{array}\right]\left[\begin{array}{c}
b \\
\alpha_{1} \\
\vdots \\
\alpha_{N}
\end{array}\right]=\left[\begin{array}{c}
0 \\
y_{1} \\
\vdots \\
y_{N}
\end{array}\right]
$$

where $K\left(\boldsymbol{x}_{i}, \boldsymbol{x}_{j}\right)=\varphi\left(\boldsymbol{x}_{i}\right)^{\mathrm{T}} \varphi\left(\boldsymbol{x}_{j}\right)$ is the kernel function, and $\gamma$ is the penalty coefficient.

(4) From the above steps, we can obtain the prediction value of downlink channel response $\tilde{h}_{\text {pra }}^{(\mathrm{DL})}$ :

$$
\tilde{h}_{\mathrm{pra}}^{(\mathrm{DL})}=f\left(\boldsymbol{x}_{N+1}\right)=\sum_{n=1}^{N} \alpha_{n} K\left(\boldsymbol{x}_{n}, \boldsymbol{x}_{N+1}\right)+b,
$$

where $\boldsymbol{x}_{N+1}=\left(h_{\mathrm{DL}(N+1)}, h_{\mathrm{DL}(N+2)}, \cdots, h_{\mathrm{DL}(N+m)}\right)$.

Through the above channel prediction method, the transmitter can obtain channel knowledge of the current time, and calculate the transmission calibration factor.

\section{Simulation results}

In this section, we present the simulation results of the proposed method. The simulation parameters are shown in Table 1.

Table 1 Simulation parameters

\begin{tabular}{ll}
\hline \multicolumn{1}{c}{ Parameter } & Value \\
\hline Maximum doppler shift & 10,100, and $150 \mathrm{~Hz}$ \\
System bandwidth & $400 \mathrm{kHz}$ \\
Number of subcarriers & 128 \\
Subcarrier interval & $3.125 \mathrm{kHz}$ \\
OFDM length & 144 \\
CP length & 16 \\
Modulation & Quadrature phase shift \\
Mismatch parameter model & $\sigma^{2}=2, \theta=\pi / 6$ \\
Channel model & Rayleigh, frequency-flat \\
Channel estimation & Ideal channel estimation \\
CP length & 16 \\
\hline
\end{tabular}

In this paper, only the performance loss caused by the calibration error during calibration is considered, and it is assumed that the free-space channel on uplink and downlink during data transmission is completely reciprocal, i.e. $h_{\mathrm{tr}}^{(\mathrm{UL})}=h_{\mathrm{tr}}^{(\mathrm{DL})}$ in Eq. (5).

The parameters for channel prediction are $Q=4, N=2$, $m=2$, and $r=10^{8}$; and the kernel function is

$$
K\left(\boldsymbol{x}_{i}, \boldsymbol{x}_{j}\right)=\exp \left(-\frac{\left\|\boldsymbol{x}_{i}-\boldsymbol{x}_{j}\right\|^{2}}{2 \sigma_{k}^{2}}\right),
$$

where $\sigma_{k}^{2}=100$ [22]. When the traditional calibration method is used for simulation, we select the channel response that is closest to the first calibration OFDM symbol on downlink as the response of uplink channel [16]; namely, the channel response for the 4th OFDM symbol on uplink is selected as the response of the uplink channel in the simulation.

Figs.4-6 show the performance of traditional calibration with the maximum Doppler shifts of 10, 100, and $150 \mathrm{~Hz}$, respectively. In these figures, the ordinate indicates the bit error rate (BER), and the abscissa indicates the ratio of bit energy to noise power spectral density, $E_{\mathrm{b}} / N_{0}$. We can see that when the maximum Doppler shift is $10 \mathrm{~Hz}$, the performance gap between the traditional calibration and the ideal calibration is small. This is because the calibration error caused by the slowly changing free-space channel is small. When the maximum Doppler shift is 100 and $150 \mathrm{~Hz}$, however, the performance loss of the traditional calibration is large because of the large calibration error caused by the time-varying channel. On the deep fading subcarrier, the preequalization factor used in the simulation compensates the deep fading while also increasing the inter-carrier interference power, so on these sub-carriers, the residual interference power appears. In high SNR regions, the noise power is low and the interference power is the main factor for the performance degradation, so the platform appears. As can be seen from Figs. 4-6, even for the ideal calibration, performance platform appears in the high SNR region. This is because the preequalization factor $\lambda_{k}=1 / h_{\mathrm{e}}^{(\mathrm{UL})}$ in Eq. (5) used for simulation is designed for static or quasi-static channels. Therefore, when the channel is varying, mutual interference occurs between adjacent sub-carriers.

Figs. 6-8 show the performance of 3 calibration methods. We can see that the performance of the prediction calibration proposed in this paper is better than the traditional calibration, especially for time-varying channels, and is very close to the performance of the ideal calibration. Thus, we can conclude that the proposed calibration algorithm is effective for timevarying channels. 


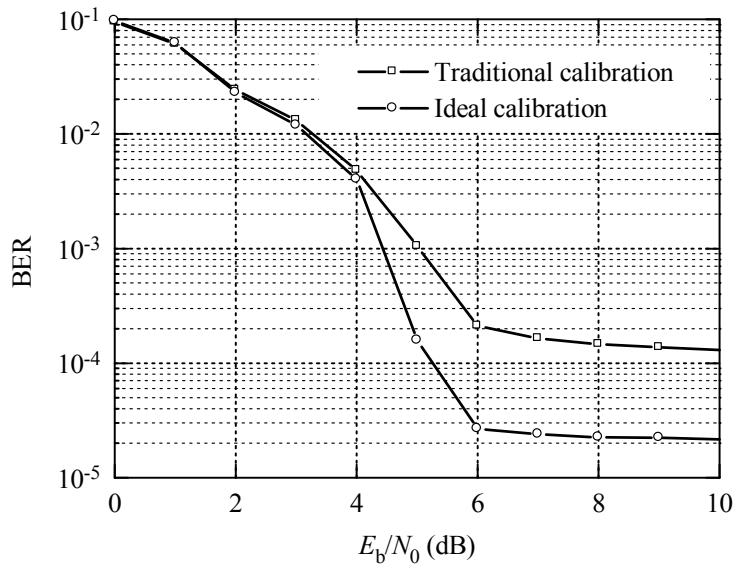

Fig. 4 Performance of traditional calibration with maximum Doppler shift of $10 \mathrm{~Hz}$

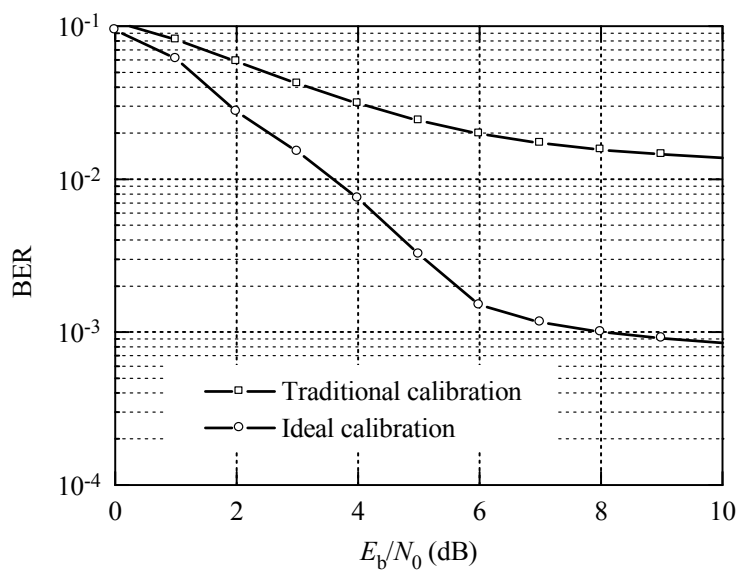

Fig. 5 Performance of traditional calibration with maximum Doppler shift of $100 \mathrm{~Hz}$

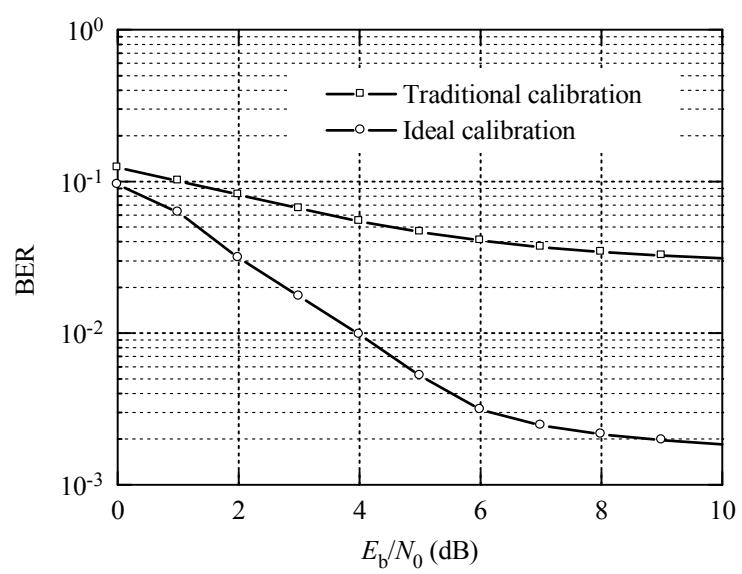

Fig. 6 Performance of traditional calibration with maximum Doppler shift of $150 \mathrm{~Hz}$

\section{Conclusion}

In TDD systems, the performance degradation of antenna calibration and pre-equalization can be caused by

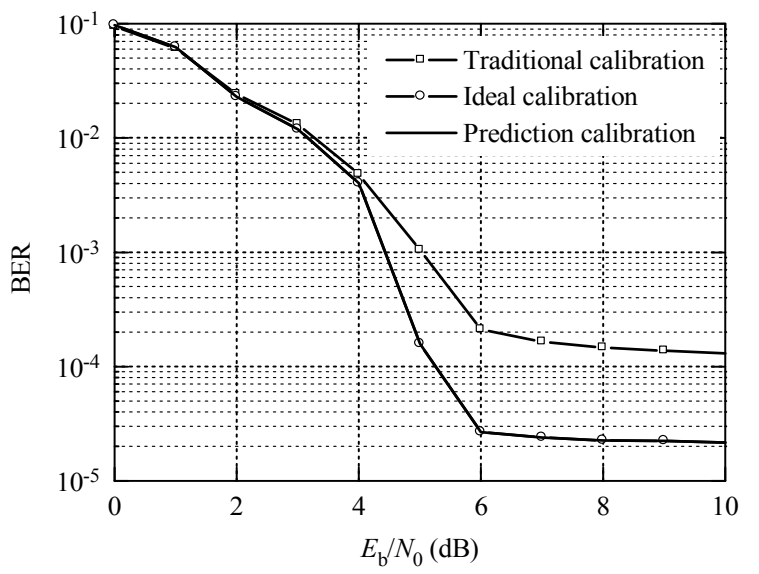

Fig. 7 Performance of calibration methods with maximum Doppler shift of $10 \mathrm{~Hz}$

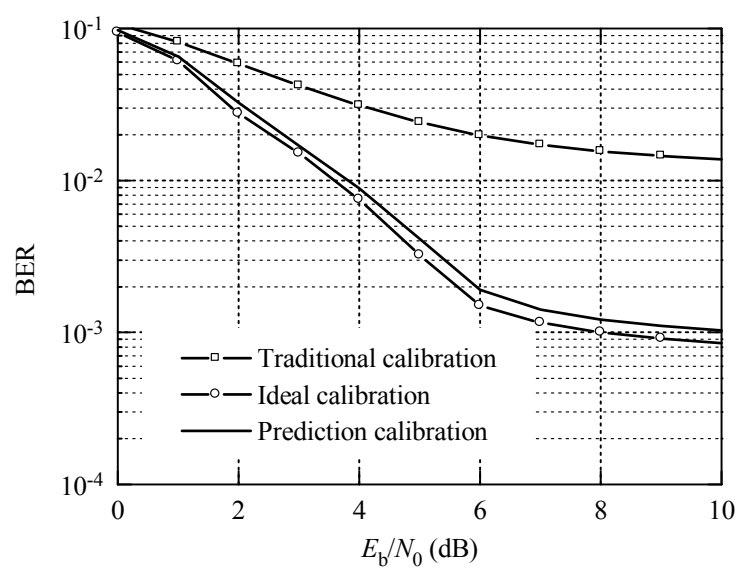

Fig. 8 Performance of calibration methods with maximum Doppler shift of $100 \mathrm{~Hz}$

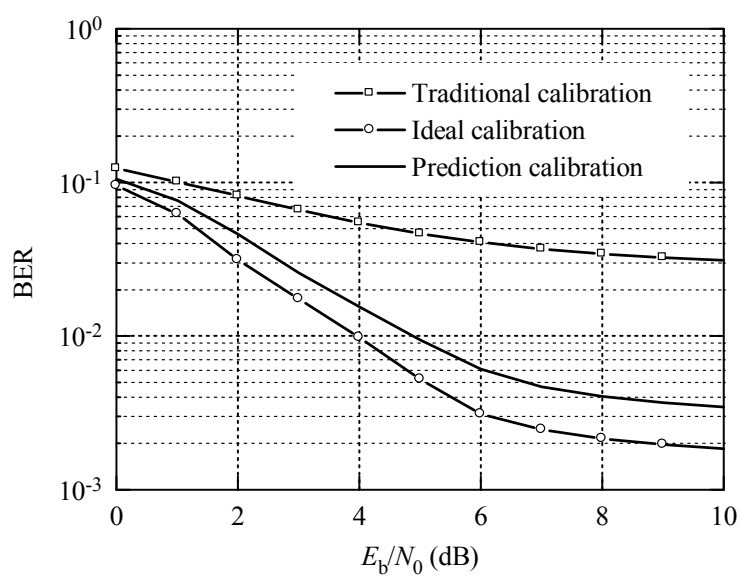

Fig. 9 Performance of calibration methods with maximum Doppler shift of $150 \mathrm{~Hz}$

time-varying channels. Especially when the channel dramatically changes, the free-space channel is no longer reciprocal. As a result, there is a large performance degradation when the traditional antenna calibration is used. The analysis and simulation in this paper 
verify the above conclusions. The proposed antenna calibration method based on channel prediction can well compensate for the performance loss caused by timevarying channels and achieve better performance.

\section{Acknowledgments}

This work was supported by the National Natural Science Foundation of China (Nos. 61032002, 61101090 and 60902026); and Chinese Important National Science \& Technology Specific Projects (No. 2011ZX03001-007-01).

\section{References}

[1] M.G. Di Benedetto, P. Manderini, An application of MMSE predistortion of OFDM system, IEEE Trans. on Commun, 1996, 44(11): 1417-1420.

[2] Y. Kim, Y. Shin, S. Im, A Memory mapping predistorter for the compensation of nonlinear distortion with memory in OFDM systems, IEEE VTC, 1999, 1: 685-689.

[3] X.Z. Xie, The 4th Generation Mobile Communication Technology Based on TDD, Bejing: Publishing House of Electronics Industry, 2005 (in Chinese).

[4] Mitsubishi Electric, Discussion on antenna calibration in TDD, In: 3GPP TSG RAN WG1 Meeting \#56, R1090563, Athens, Greece, Feb. 9-13, 2009: 1-6.

[5] Alcatel-Lucent Shanghai Bell, An over-the-aircalibration scheme for ZF based SU/MU-MIMO, In: 3GPP TSG RAN1\#58, R1-093369, Shenzhen, China, August 24-28, 2009: 1-4.

[6] Alcatel-Lucent Shanghai Bell, Channel reciprocity modeling and performance evaluation, In: 3GPP TSG RAN1\#59bis, R1-100426, Valencia, Spain, January 1822, 2010: 1-10.

[7] Y.S. Song, D.S. Kwon, Computationally efficient compensations for some smart antenna systems, IEEE VTC, 2004, 7: 4786-4788.

[8] Alcatel-Lucent Shanghai Bell, Channel reciprocity modeling and performance evaluation, In: 3GPP TSG RAN1 \#56, R1-094622, Jeju, Korea, Nov. 9-13, 2009.

[9] Y. Hara, Y. Yano, H. Kubo, Antenna array calibration using frequency selection in OFDMA/TDD systems, In: Proceedings of IEEE Globecom, New Orleans, LA, USA, Nov. 30-Dec. 4, 2008: 1-5.

[10] A. Bourdoux, B. Come, N. Khaled, Non-reciprocity transceivers in OFDM/SCDMA system: impact and mitigation, In: Proceedings of Radio and Wireless Conference 2003 (RAWCON'03), Boston, Massachusetts, USA, Aug. 10-13, 2003: 183-186

[11] M. Guillaud, D. Slock, R. Knopp, A practical method for wireless channel reciprocity exploitation through relative calibration. In: Proc. of ISSPA 2005, Sydney, Australia, Sep. 2005: 403-406.

[12] F. Kaltenberger, H. Jiang, M. Guillaud, et al., Relative channel reciprocity calibration in MIMO/TDD systems, In: Proceedings of Future Network and Mobile Summit 2010, Florence, Italy, Jun. 16-18, 2010: 1-10.

[13] W.J. Welch, Reciprocity theorems for electromagnetic fields whose time dependence is arbitrary, IRE Trans. Antennas and Propagation, 1960, 8(1): 68-73.

[14] Alcatel-Lucent Shanghai Bell, Channel reciprocity modeling and performance evaluation, In: 3GPP TSG RAN1\#59, R1-094622, Jeju, Korea, Nov. 9-13, 2009:1-10.

[15] CMCC, Antenna calibration requirement for CoMP, In: IMT-A_LTE+\#19, IMT-A-TECH-08525, Beijing, China, Oct. 29, 2009.

[16] Qualcomm Europe, Calibration procedures for TDD beamforming, In: 3GPP TSG RAN1 \#51, R1-080494, Sevilla, Spain, Jan. 14-18, 2008: 1-6.

[17] Huawei, Hardware calibration requirement for dual layer beamforming, In: 3GPP TSG RAN WG1 Meeting \#57, R1092359, Los Angeles, USA, Jun. 29-Jul. 3, 2009: 1-10.

[18] M. Sanchez-Fernandez, A. Lozano, Doppler sensitivity of link reciprocity in TDD MIMO systems, In: Proceedings of IEEE Globecom, St. Louis, MO, Dec. 2, 2005 : 3073-3076.

[19] A. Duel-Hallen, Fading channel prediction for mobile radio adaptive transmission system, Proceedings of the IEEE, 2007, 95(12): 2299-2313.

[20] E. Karami, M. Silva, Blind multi-input-multi-output channel tracking using decision-directed maximumlikelihood estimation, IEEE Transactions on Vehicular Technology, 2007, 56(3): 1447-1454.

[21] J.A.K. Suykens, J. Vandewalle, Recurrent least squares support vector machines, IEEE Trans on Circuits and Systems-I: Fundamental Theory and Applications, 2000, 47(7): 1109-1114.

[22] L. Zhu, Channel Reciprocity Compensation Method in Time-Varying TDD-MIMO System Based on SVM Channel Prediction [Dissertation], Xi'an: XiDian University, 2011 (in Chinese). 